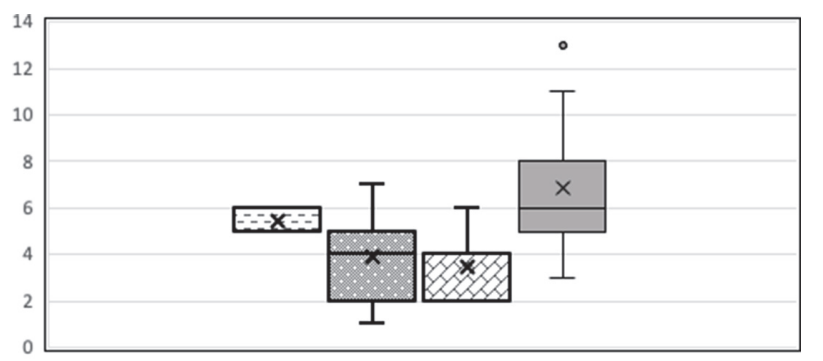

Q Colonic Series $\square$ Right $\boldsymbol{Q}$ Left $\square$ Total

Abstract PTU-62 Figure 1 Biopsies taken in those diagnosed with MC

The diagnosis of MC was made in 20 cases. The age distribution was 30 to 80 (median 67.5) with male to female ratio 3:7. All had biopsies taken from both right and left colon amounting to 3 to 13 biopsies in total. The most common biopsy pattern in those diagnosed with MC were 5-6 biopsies taken as colonic series (25\%) or 2-4 biopsies taken from both right and left colon (45\%). Distribution in number of biopsies is shown in Table 1.

Conclusion There was a large variation in the sites and number of biopsies taken to investigate for MC reflecting the lack of established guidance on biopsy protocol. Excessive biopsy samples have negative impacts on our green footprint, increase workload for histopathology colleagues and potential risk in bleeding and perforation risk for patients.

We propose that the BSG should implement MC diagnostic biopsy protocol for standardisation of our practice. From the results of our data we advocate for either 5-6 biopsies as a colonic series or 2-4 biopsies to be taken from both right and left colon. Biopsies should not be taken from the rectum in a macroscopically normal colon.

*Multiple biopsies ordered from proximal to distal colon

\section{PTU-63 SEMI-AUTOMATED ANNOTATION TOOL OUTPERFORMS MEDICAL STUDENTS AND IS COMPARABLE TO CLINICAL EXPERTS FOR POLYP DETECTION}

${ }^{1}$ Tom Eelbode, ${ }^{2}$ Omer Ahmad* ${ }^{3}$ Pieter Sinonquel, ${ }^{2}$ Timon B Kocadag, ${ }^{2}$ Neil Narayan, ${ }^{2}$ Nikita Rana, ${ }^{1}$ Ir Frederik Maes, ${ }^{2}$ Laurence B Lovat, ${ }^{3}$ Raf Bisschops. ${ }^{1}$ ESAT/PSI, KU Leuven, Leuven, Belgium; ' Wellcome/EPSRC Centre for Interventional and Surgical Sciences (WEISS), University College London Hospitals, London, UK; ${ }^{3}$ Gastroenterology and hepatology, UZ Leuven, Leuven, Belgium

\subsection{6/gutjnl-2021-BSG.136}

Introduction Expert labelling of each frame in a polyp video is the most robust way for constructing a training set for deep learning, but this is very time-consuming and currently represents a major barrier for widespread implementation of AI in endoscopy. In this study, two alternative approaches are evaluated, an innovative semi-automated labelling tool and trained medical students providing annotations.

Methods 20 unique polyp white light videos containing 6282 frames (14 adenomas and 6 sessile serrated lesions confirmed by histopathology, mean size $7 \mathrm{~mm}$, Olympus) were annotated with bounding boxes by a clinical expert. These annotations are used as the gold standard for comparison. Two cheaper annotation methods were then applied to evaluate their validity and relative performance: (1) a semi-automated labelling technique - this tool only requires 3 manually annotated

\begin{tabular}{lllll}
\multicolumn{5}{l}{ Abstract PTU-63 Table 1 } \\
& Sensitivity & PPV & Adjudicated PPV & Time (mins) \\
\hline Student 1 & $74,38 \pm 27,30$ & $88,52 \pm 30,51$ & $89,92 \pm 15,34$ & 264 \\
Student 2 & $63,08 \pm 20,27$ & $94,69 \pm 22,30$ & $95,00 \pm 07,47$ & 1208 \\
Student 3 & $66,97 \pm 27,37$ & $94,77 \pm 22,32$ & $95,00 \pm 12,30$ & 234 \\
Semi-automated & $94,40 \pm 06,22$ & $97,17 \pm 05,87$ & $98,97 \pm 14,04$ & 25 \\
\hline
\end{tabular}

video frames, from which a representation of the polyp is learned and transferred automatically to all the other frames in the video; (2) independent manual labelling of each video by three medical students - following a training module with polyp images and videos.

Results The mean and standard deviation of the frame-level sensitivity, positive predictive value (PPV) and adjudicated PPV (for borderline low-quality frames) over all videos are provided in table 1 . The semi-automated method significantly outperforms all three students on sensitivity and annotation time (paired t-test, p-value < 0.05), while also achieving the highest value for PPV, both before and after adjudication.

Conclusions A semi-automated labelling tool is a faster, more efficient and valid approach for polyp detection. It outperforms three medical students, specifically trained for polyp recognition and is comparable to clinical expert performance.

\section{PTU-64 SALINE IMMERSION THERAPEUTIC ENDOSCOPY FACILITATED ENDOSCOPIC SUBMUCOSAL DISSECTION OF AN ILEAL LIPOMA DURING ENTEROSCOPY}

${ }^{1}$ Alexandros Skamnelos, ${ }^{1}$ Alberto Murino, ${ }^{1,2}$ Rui de Sousa Magalhães, ${ }^{1}$ Rocio Chacchi Cahuin*, ${ }^{3}$ TuVinh Luong, ${ }^{1}$ Regina Raymond, ${ }^{1}$ Nikolaos Lazaridis, ${ }^{1}$ Edward J Despott. 'Royal Free Unit for Endoscopy, The Royal Free Hospital and University College London (UCL) Institute for Liver and Digestive Health, London, UK; ${ }^{2}$ Gastroenterology Department, Hospital Senhora da Oliveira - Guimarães, Life and Health Sciences Research Institute (ICVS), School of Medicine, University of Minho, Braga, Portugal; ${ }^{3}$ Department of Cellular Pathology, The Royal Free Hospital and University College London (UCL) Institute for Liver and Digestive Health, London, UK

\subsection{6/gutjnl-2021-BSG.137}

Introduction Small bowel lipomas are benign, usually innocuous lesions which more frequently affect the terminal ileum (TI). They are usually asymptomatic but may give rise to obstructive symptoms and gastrointestinal bleeding, especially if $\geq 2 \mathrm{~cm}$ in diameter. Although in such cases, surgery has been the mainstay of care, endoscopic resection is being increasingly employed. Endoscopic submucosal dissection (ESD) is emerging as a safe and definitive endotherapeutic, minimally invasive strategy for such lesions.

Methods A 69-year-old man with ischaemic heart disease and cerebrovascular disease was referred to our tertiary institution for endoscopic resection of a large, symptomatic, intussuscepting lipoma of the TI. At retrograde double-balloon enteroscopy (DBE), under conscious sedation, a $35 \mathrm{~mm}$ semipedunculated submucosal lesion was identified within the TI, about $5 \mathrm{~cm}$ proximal to the ileocecal valve.

Results Endoscopic morphology was pathognomonic of a lipoma. The lesion's size and its location within the narrow lumen of the TI precluded safe snare resection and we therefore proceeded with ESD. After injection of submucosal-lifting-solution (succinylated gelatin, indigo carmine $(0.05 \%)$ and dilute epinephrine $(1: 100,000))$, the stalk was dissected using 